\title{
Studying the Interrelationship amongst Various Metrics used by Health Care Facilities in India
}

\author{
Remica Aggarwal \\ School of Business, University of Petroleum \& \\ Energy Studies, Dehradun, India
}

\author{
Veena Aggarwal \\ Recventures Education Services Private Limited, \\ Delhi, India
}

\begin{abstract}
India's health care sector provides a wide range of quality of care from globally acclaimed hospitals to facilities that deliver care of unacceptably low quality. Technology is the best way to achieve the vision of a connected healthcare ecosystem . Present research work tries to explore various metrics commonly used by health care facilities or centers in developing countries like India. It further tries to find the inter-relationship amongst them using ISM methodology.
\end{abstract}

\section{Keywords}

Health care metrics, ISM methodology, health care facility

\section{INTRODUCTION}

Health care is one of the fastest growing sector in India and has made substantial progress, especially in the last decade. Between 2000 and 2014, there was a 370\% increase in health expenditure. $^{1}$ Historically , health care delivery in independent India has been under the purview of government. Indian government is striving hard to provide world class and affordable health services to its citizens. On the policy side, Indian government has made a bold commitment to achieve universal health coverage through Ayushman Bharat (AB) which aims to provide affordable health care to entire population and reduce expenses on health care. On the implementation side, improving healthcare infrastructure takes time and money. There is an increasing emphasis from government to reduce drug prices and make medicines more affordable. For example, some of the critical dug for cancer treatments are now $86 \%$ cheaper whereas prices for diabetes drugs are down by $42 \%$.

There is also a shift in government role in Indian healthcare from that of a provider to an insurer. With Union budget 2017 , the Indian government has pushed to make Aadhar card a health identifier for basic health services in the country. Another important action was the launch of insurance plan named Ayushman Bharat to provide the insurance cover to 10 crores families in India . Further, under the guidance of NITI Aayog, approximately 3,073 crore INR has been set to be allocated to create a digital economy with emerging technologies such as artificial intelligence (AI), the Internet of things (IoT), block chain and 3D printing, which are necessary to build a modern technology landscape in health care delivery. These technologies can reduce the burden and improve productivity greatly by minimizing human interventions in electronic medical records (EMR) / enterprise resource planning (ERP) . One of the striking features of India's health care sector is the range of quality in available services. India is home to global leaders in innovation and quality of health care such as the Narayana Hospitals, known for providing high-quality cardiovascular surgery at low cost, and the Aravind Eye Care System, whose hospitals provide a high volume of cataract surgery, as well as globally renowned medical teaching institutions such as the All India Institute of
Medical Sciences, in New Delhi. Efforts to improve the quality of health care services in low-resource settings, including India, have typically focused on structural constraints. Objectives of paper are therefore to explore various KPIs and metrics used by health care facilities in developing countries like India for improving their performance, productivity and quality of health care services thereby increasing their patient traffic. Afterwards, it also tries to study the hierarchical relationships amongst them using the Interpretive Structural Modeling methodology. Paper is arranged as follows: Section 2 briefly details the literature available on the given topic and track different metrics as per literature. Section 3 explains the ISM methodology and explains it through the case example in section 4. Section 5 briefly provides the managerial implications on the given topic.

\section{LITERATURE REVIEW HEALTH} CARE AND ITS ASSOCIATED METRICS This section focuses on the literature review on various health care metrics described in literature. Authors have used keywords such as "health care metrics", "KPIs used by health facilities", "health-care facilities in India", " metrics for improving health care" etc. over the google search engine, Mendeley research database software to collect the relevant literature. Table 1 . below lists the various commonly used metrics by health care facilities or organizations in developing countries such as India .

Table 1. Important metrics and sub metrics for health care facility [1-5]

\begin{tabular}{|c|c|c|c|c|}
\hline $\begin{array}{c}\text { S. } \\
\text { No } \\
\text {. }\end{array}$ & $\begin{array}{c}\text { Metrics \& } \\
\text { their } \\
\text { descriptio } \\
\text { n }\end{array}$ & Sub metrics & $\begin{array}{c}\text { Descriptio } \\
\text { n }\end{array}$ & Purpose \\
\hline \multirow[t]{3}{*}{1.} & \multirow[t]{3}{*}{$\begin{array}{l}\text { Volume } \\
\text { metrics : } \\
\text { This data } \\
\text { refers to } \\
\text { the flow of } \\
\text { patients to } \\
\text { your } \\
\text { facility. }\end{array}$} & $\begin{array}{l}\text { Number of } \\
\text { patients } \\
\text { (NOP) }\end{array}$ & $\begin{array}{c}\text { This } \\
\text { includes } \\
\text { number of } \\
\text { patients } \\
\text { department } \\
\text { wise as } \\
\text { well as } \\
\text { specialty } \\
\text { wise }\end{array}$ & \multirow{3}{*}{$\begin{array}{c}\text { Tracking } \\
\text { these } \\
\text { metrics } \\
\text { allows you } \\
\text { to determine } \\
\text { if current } \\
\text { marketing } \\
\text { and } \\
\text { business } \\
\text { outreach } \\
\text { and } \\
\text { developmen } \\
\text { t is as } \\
\text { effective as } \\
\text { the } \\
\text { projections } \\
\text { made ; to } \\
\text { reassess }\end{array}$} \\
\hline & & $\begin{array}{c}\text { Time of } \\
\text { appointment } \\
\text { and length of } \\
\text { visit (TOA) }\end{array}$ & $\begin{array}{c}\text { This } \\
\text { includes } \\
\text { appointmen } \\
\mathrm{t} \text { time and } \\
\text { duration of } \\
\text { visit }\end{array}$ & \\
\hline & & $\begin{array}{c}\text { Number of } \\
\text { inbound }\end{array}$ & $\begin{array}{c}\text { This } \\
\text { includes }\end{array}$ & \\
\hline
\end{tabular}




\begin{tabular}{|c|c|c|c|c|}
\hline & & $\begin{array}{c}\text { referrals } \\
\text { (NIR) }\end{array}$ & $\begin{array}{l}\text { number of } \\
\text { inbound } \\
\text { referrals }\end{array}$ & $\begin{array}{c}\text { current } \\
\text { service } \\
\text { offerings } \\
\text { and } \\
\text { determine if } \\
\text { you need to } \\
\text { invest in } \\
\text { other areas } \\
\text { of expertise } \\
\text { or improve } \\
\text { and expand } \\
\text { the existing } \\
\text { lines of } \\
\text { business. }\end{array}$ \\
\hline \multirow[t]{3}{*}{2.} & \multirow{3}{*}{$\begin{array}{l}\text { Revenue } \\
\text { leakage } \\
\text { metrics : } \\
\text { This refers } \\
\text { to the data } \\
\text { related to } \\
\text { the flow of } \\
\text { patients } \\
\text { through } \\
\text { your } \\
\text { facility } \\
\text { with lost } \\
\text { income } \\
\text { opportuniti } \\
\text { es }\end{array}$} & $\begin{array}{c}\text { Cancelled } \\
\text { appointments } \\
\text { (CA) }\end{array}$ & $\begin{array}{l}\text { This } \\
\text { includes } \\
\text { cancelled } \\
\text { appointmen } \\
\text { ts due to } \\
\text { patients not } \\
\text { showing up }\end{array}$ & \multirow{3}{*}{$\begin{array}{c}\text { Tracking } \\
\text { these } \\
\text { metrics } \\
\text { helps you } \\
\text { gauge the } \\
\text { effectivenes } \\
\text { s of your } \\
\text { patient } \\
\text { appointment } \\
\text { and } \\
\text { appointment } \\
\text { reminder } \\
\text { systems. } \\
\text { These } \\
\text { metrics also } \\
\text { provide } \\
\text { similar } \\
\text { insight into } \\
\text { the need to } \\
\text { expand } \\
\text { current } \\
\text { business } \\
\text { offerings. }\end{array}$} \\
\hline & & $\begin{array}{l}\text { Number of } \\
\text { outbound } \\
\text { referrals } \\
\text { (NOR) }\end{array}$ & $\begin{array}{l}\text { This } \\
\text { includes } \\
\text { tracking the } \\
\text { number of } \\
\text { outbound } \\
\text { referrals } \\
\text { due to } \\
\text { unavailabili } \\
\text { ty and un-- } \\
\text { supported } \\
\text { specializati } \\
\text { ons }\end{array}$ & \\
\hline & & $\begin{array}{l}\text { Rescheduled } \\
\text { appointment( } \\
\text { RA) }\end{array}$ & $\begin{array}{c}\text { This } \\
\text { include } \\
\text { cancelled or } \\
\text { rescheduled } \\
\text { appointmen } \\
\text { ts due to } \\
\text { unavailabili } \\
\text { ty of } \\
\text { provider }\end{array}$ & \\
\hline \multirow[t]{3}{*}{3.} & \multirow{3}{*}{$\begin{array}{c}\text { Utilization } \\
\text { metrics : } \\
\text { This data } \\
\text { refers to } \\
\text { the patient } \\
\text { traffic and } \\
\text { consumpti } \\
\text { on of } \\
\text { resources } \\
\text { generated } \\
\text { by each } \\
\text { department } \\
\text { specializati } \\
\text { on, and } \\
\text { service } \\
\text { provider }\end{array}$} & $\begin{array}{l}\text { Appointment } \\
\text { s completed } \\
\text { per doctor } \\
\text { (ACD) }\end{array}$ & $\begin{array}{c}\text { This } \\
\text { includes } \\
\text { number of } \\
\text { appointmen } \\
\text { ts seen or } \\
\text { handles by } \\
\text { each } \\
\text { available } \\
\text { doctor }\end{array}$ & \multirow{3}{*}{$\begin{array}{c}\text { More } \\
\text { specialized } \\
\text { than the } \\
\text { volume or } \\
\text { revenue } \\
\text { leakage } \\
\text { metrics, } \\
\text { drilling data } \\
\text { down on the } \\
\text { dashboard } \\
\text { to this level } \\
\text { allows you } \\
\text { to determine } \\
\text { your most } \\
\text { requested } \\
\text { services and } \\
\text { expand your } \\
\text { capacity to } \\
\text { meet the } \\
\text { demographi } \\
\text { cs and } \\
\text { demands of }\end{array}$} \\
\hline & & $\begin{array}{l}\text { Surgeries } \\
\text { performed } \\
\text { per surgeon } \\
\quad(\text { SPS })\end{array}$ & $\begin{array}{c}\text { This } \\
\text { includes the } \\
\text { number of } \\
\text { surgeries } \\
\text { performed } \\
\text { per surgeon }\end{array}$ & \\
\hline & & $\begin{array}{l}\text { Appointment } \\
\text { s scheduled } \\
\text { per } \\
\text { department } \\
\text { and } \\
\text { specializatio }\end{array}$ & $\begin{array}{c}\text { This } \\
\text { includes } \\
\text { number of } \\
\text { appointmen } \\
\text { ts } \\
\text { scheduled }\end{array}$ & \\
\hline
\end{tabular}

\begin{tabular}{|c|c|c|c|c|}
\hline & & $\mathrm{n}(\mathrm{ASD} / \mathrm{S})$ & $\begin{array}{l}\text { department } \\
\text { wise as } \\
\text { well as } \\
\text { specializati } \\
\text { on wise }\end{array}$ & $\begin{array}{c}\text { your patient } \\
\text { base. It also } \\
\text { reflects } \\
\text { whether all } \\
\text { resources at } \\
\text { your } \\
\text { hospital are } \\
\text { efficiently } \\
\text { utilized or } \\
\text { not. }\end{array}$ \\
\hline \multirow[t]{3}{*}{4.} & \multirow{3}{*}{$\begin{array}{c}\text { Quality } \\
\text { metrics: } \\
\text { This data } \\
\text { relates to } \\
\text { the } \\
\text { effectivene } \\
\text { ss of your } \\
\text { quality, } \\
\text { safety, and } \\
\text { access } \\
\text { initiatives. } \\
\text { It is the } \\
\text { most } \\
\text { extensive } \\
\text { data-set } \\
\text { that will be } \\
\text { tracked on } \\
\text { your } \\
\text { dashboard }\end{array}$} & $\begin{array}{c}\text { Employee } \\
\text { Satisfaction } \\
\text { (ES) }\end{array}$ & $\begin{array}{c}\text { This } \\
\text { calculates } \\
\text { degree / } \\
\text { level of } \\
\text { employee } \\
\text { satisfaction }\end{array}$ & \multirow{3}{*}{$\begin{array}{c}\text { These } \\
\text { metrics } \\
\text { track the } \\
\text { impact of } \\
\text { your } \\
\text { services on } \\
\text { the patients } \\
\text { and your } \\
\text { employees. } \\
\text { There's no } \\
\text { point in } \\
\text { increasing } \\
\text { traffic } \\
\text { through } \\
\text { your facility } \\
\text { if your } \\
\text { employees } \\
\text { aren't } \\
\text { providing } \\
\text { high quality } \\
\text { healthcare, } \\
\text { and these } \\
\text { set of } \\
\text { metrics help } \\
\text { you control } \\
\text { quality of } \\
\text { healthcare } \\
\text { delivery. }\end{array}$} \\
\hline & & $\begin{array}{c}\text { Patient } \\
\text { Satisfaction } \\
\text { and } \\
\text { Engagement } \\
\text { (PSE) }\end{array}$ & $\begin{array}{c}\text { This } \\
\text { includes } \\
\text { degree or } \\
\text { level of } \\
\text { satisfaction } \\
\text { and } \\
\text { engagement } \\
\text { of patient }\end{array}$ & \\
\hline & & $\begin{array}{c}\text { Post- } \\
\text { Treatment } \\
\text { metrics } \\
(\mathrm{PTM})\end{array}$ & $\begin{array}{c}\text { This } \\
\text { includes } \\
\text { metrics } \\
\text { such as } \\
\text { Readmissio } \\
\text { n rates for } \\
\text { specific } \\
\text { diseases } \\
\text { (e.g. heart } \\
\text { failure, } \\
\text { pneumonia, } \\
\text { etc.), } \\
\text { Clinical } \\
\text { outcome } \\
\text { rate } \\
\text { statistics, } \\
\text { Occurrence } \\
\text { of hospital } \\
\text { acquired } \\
\text { infections, } \\
\text { Clinical } \\
\text { error ratios } \\
\text { and } \\
\text { outpatient } \\
\text { wait time }\end{array}$ & \\
\hline \multirow[t]{2}{*}{5.} & \multirow{2}{*}{$\begin{array}{c}\text { Financial } \\
\text { metrics : } \\
\text { This is the } \\
\text { data that is } \\
\text { more } \\
\text { finely } \\
\text { tuned and } \\
\text { focused on } \\
\text { the fiscal } \\
\text { performan } \\
\text { ce of your } \\
\text { healthcare } \\
\text { facility }\end{array}$} & $\begin{array}{c}\text { Revenue } \\
\text { generated per } \\
\text { doctor } \\
\text { (RGD) }\end{array}$ & $\begin{array}{l}\text { This } \\
\text { includes the } \\
\text { amount of } \\
\text { revenue } \\
\text { generated } \\
\text { by each } \\
\text { doctor in } \\
\text { the health } \\
\text { care facility }\end{array}$ & \multirow{2}{*}{$\begin{array}{c}\text { When } \\
\text { drilling } \\
\text { down into } \\
\text { this data and } \\
\text { cross- } \\
\text { referencing } \\
\text { it against } \\
\text { the } \\
\text { utilization } \\
\text { metrics } \\
\text { outlined } \\
\text { above, you } \\
\text { and the } \\
\text { decision } \\
\text { makers of }\end{array}$} \\
\hline & & $\begin{array}{c}\text { Revenue } \\
\text { generated by } \\
\text { department } \\
\text { (RGDe) }\end{array}$ & $\begin{array}{l}\text { This } \\
\text { includes the } \\
\text { amount of } \\
\text { revenue } \\
\text { generated } \\
\text { by each }\end{array}$ & \\
\hline
\end{tabular}




\begin{tabular}{|c|c|c|c|c|}
\hline & & & $\begin{array}{l}\text { department } \\
\text { in the } \\
\text { health care } \\
\text { facility }\end{array}$ & \multirow{2}{*}{$\begin{array}{c}\text { your } \\
\text { organization } \\
\text { can better } \\
\text { gauge how } \\
\text { to increase } \\
\text { profitability } \\
\text { by } \\
\text { expanding } \\
\text { your most } \\
\text { profitable } \\
\text { channels of } \\
\text { business. }\end{array}$} \\
\hline & & $\begin{array}{c}\text { Revenue } \\
\text { generated by } \\
\text { specializatio } \\
\text { n (RGS) }\end{array}$ & $\begin{array}{c}\text { This } \\
\text { includes the } \\
\text { amount of } \\
\text { revenue } \\
\text { generated } \\
\text { as per } \\
\text { doctor / } \\
\text { department } \\
\text { specializati } \\
\text { on }\end{array}$ & \\
\hline \multirow[t]{3}{*}{6.} & \multirow[t]{3}{*}{$\begin{array}{c}\text { Miscellane } \\
\text { ous } \\
\text { metrics }\end{array}$} & $\begin{array}{c}\text { Average } \\
\text { Hospital Stay } \\
\text { (AHS) }\end{array}$ & $\begin{array}{l}\text { Evaluate } \\
\text { the amount } \\
\text { of time } \\
\text { your } \\
\text { patients are } \\
\text { staying }\end{array}$ & \multirow{3}{*}{$\begin{array}{l}\text { These } \\
\text { metrics are } \\
\text { important to } \\
\text { get an } \\
\text { overview of } \\
\text { the } \\
\text { performance } \\
\text { of the health } \\
\text { care facility. }\end{array}$} \\
\hline & & $\begin{array}{l}\text { Treatment } \\
\text { Costs (TC) }\end{array}$ & $\begin{array}{c}\text { Calculate } \\
\text { how much } \\
\text { a patient } \\
\text { costs to } \\
\text { your } \\
\text { facility }\end{array}$ & \\
\hline & & $\begin{array}{l}\text { Patient Wait } \\
\text { Time (PWT) }\end{array}$ & $\begin{array}{c}\text { Monitor } \\
\text { waiting } \\
\text { times to } \\
\text { increase } \\
\text { patient } \\
\text { satisfaction }\end{array}$ & \\
\hline
\end{tabular}

\section{INTERPRETIVE STRUCTURAL MODELLING METHODOLOGY}

Interpretive Structural Modeling or ISM first proposed by [6] is a computer assisted learning process that enables the researcher to develop a map of the complex relationships between the many elements involved in a complex situation. In this technique a set of unique interrelated variables are structured in the form of a hierarchy graph called the diagraph. Its steps are as follows: Firstly, identify the relevant elements and establish a contextual relationship amongst them. Thereafter, develop a structural self -interaction matrix (SSIM) using $\mathrm{V}, \mathrm{A}, \mathrm{X} \& \mathrm{O}$ where the symbols have the following meanings i.e. $\mathrm{V}$ for the relation from $\mathrm{i}$ to $\mathrm{j}$ but not in both directions; A for the relation from $\mathrm{j}$ to $\mathrm{i}$ but not in both directions; $X$ for both direction relations from $i$ to $j$ and $j$ to $i$; and $\mathrm{O}$ if the relation between the variables does not appear valid. Using SSIM, initial reachability matrix can be formed which has all values in binary form. A final reachability matrix is formed after removing transitivity from initial reachability matrix. From the final reachability matrix, the reachability set and antecedent set for each criterion and for each element is found. The element for which the reachability and intersection sets are the same is the top-level element. At every iteration a top level element is identified which is removed in the next iteration. After all the elements have been identified at different level of hierarchy, a Mic-Mac analysis (based on the driving power and dependence power) and a diagraph can be formed.

\section{DEVELOPMENT OF ISM MODEL}

This section develops the ISM model for studying the interrelationships amongst the metrics in building and construction industry. The 20 metrics considered are Number of patients (NOP); Time of appointment and length of visit (TOA); Number of inbound referrals (NIR) ; Cancelled appointments (CA) ; Number of outbound referrals (NOR) ; Rescheduled appointments (RA) ; Appointments completed per doctor (ACD) ; Surgeries performed per surgeon (SPS) ; Appointments scheduled per department and specialization $(\mathrm{ASD} / \mathrm{S})$; Employee satisfaction (ES) ; Patient satisfaction and engagement (PSE) ; Post-treatment metrics (PTM) ; Revenue generated per doctor/surgeon (RGDS) ; Revenue generated by department (RGDe) ; Revenue generated by specialization (RGS) ; Average hospital stay (AHS) ; Treatment costs (TC); Patient wait time (PWT) .

\subsection{Construction of Structural Self - Interaction Matrix (SSIM)}

This matrix gives the pair-wise relationship between two variables i.e. $\mathrm{i}$ and $\mathrm{j}$ based on VAXO. SSIM has been presented below in Fig 1.

\section{Explanation:}

Number of inbound and outbound referrals lead to increase in number of patients . Patient satisfaction and engagement also increase number of patients . Number of patients lead to revenue generated by department, revenue generated by doctor or specialization. Patient wait time is associated with time of appointment and length of visit . Employee satisfaction may lead to inbound referrals . Similarly, patient satisfaction may lead to outbound referrals . Treatment costs may lead to revenue generated by department and as per specialization. Surgeries performed per surgeon leads to revenue generated by doctor / surgeon and specialization as well as department . Patient wait time may lead to cancelled appointments . Number of patients and time of appointment leads to appointments scheduled per department and rescheduled appointments also in many cases. Patient wait time leads to cancelled appointments and rescheduled appointments . Time of appointment also leads to increase in number of patients as usually patients avoid going in peak hours otherwise urgent. Employee satisfaction also lead to more referrals and hence may indirectly lead to increase in number of patients. Similarly, is the case with patient satisfaction and engagement and patient wait time. Number of inbound and outbound referrals may decide on may depend on time of appointment and length of visit. Number of inbound referrals may determine the post treatment metrics and patient wait time as it is quite possible that these referrals may not have to wait for long hours . Some outbound referrals may also cancel or reschedule their appointments . Outbound referrals could also be a part of surgeries performed by surgeon , appointments completed per doctor etc. Lesser the hospital re-admission rates, more could be the outbound referrals as this shows the positive and efficient services rendered by hospital. Treatment costs also determine appointments completed by doctor as high treatment costs may not be affordable by every patient. Long wait times negatively affects the revenue generation. Higher treatment costs lead to lesser wait time. 
4.2 Construction of Initial Reachability

Matrix and final reachability matrix

The SSIM has been converted in to a binary matrix called the

initial reachability matrix shown in fig. 2 by substituting V, A,

Fig 1: Structural Self- Interaction Matrix

\begin{tabular}{|c|c|c|c|c|c|c|c|c|c|c|c|c|c|c|c|c|c|c|c|}
\hline Metric & & 1 & 2 & 3 & 4 & 5 & 6 & 7 & 8 & 9 & 10 & 11 & 12 & 13 & 14 & 15 & 16 & 17 & 18 \\
\hline & & NOP & $\begin{array}{l}\mathrm{T} \\
\mathrm{O} \\
\mathrm{A}\end{array}$ & NIR & $\begin{array}{l}\mathrm{C} \\
\mathrm{A}\end{array}$ & $\begin{array}{l}\mathrm{N} \\
\mathrm{O} \\
\mathrm{R}\end{array}$ & $\begin{array}{l}\mathrm{R} \\
\mathrm{A}\end{array}$ & $\begin{array}{l}\text { A } \\
\text { C } \\
\text { D }\end{array}$ & $\begin{array}{c}\text { SP } \\
\text { S }\end{array}$ & $\begin{array}{c}\text { AS } \\
\text { D }\end{array}$ & ES & $\begin{array}{c}\text { PS } \\
\text { E }\end{array}$ & $\begin{array}{l}\text { PT } \\
\text { M }\end{array}$ & $\begin{array}{c}\mathrm{R} \\
\mathrm{G} \\
\mathrm{DS}\end{array}$ & $\begin{array}{c}\mathrm{R} \\
\mathrm{G} \\
\mathrm{De}\end{array}$ & $\begin{array}{l}\mathrm{R} \\
\mathrm{G} \\
\mathrm{S}\end{array}$ & $\begin{array}{l}\mathrm{A} \\
\mathrm{H} \\
\mathrm{S}\end{array}$ & $\begin{array}{l}\mathrm{T} \\
\mathrm{C}\end{array}$ & $\begin{array}{c}\mathrm{P} \\
\mathrm{W} \\
\mathrm{T}\end{array}$ \\
\hline 1 & NOP & & $\mathrm{A}$ & $\bar{A}$ & $\bar{A}$ & $\bar{A}$ & $\overline{\mathrm{A}}$ & A & $\mathrm{O}$ & $X$ & $\mathrm{~A}$ & $\mathrm{~A}$ & $\mathrm{~A}$ & $\mathrm{~V}$ & $\mathrm{~V}$ & $\mathrm{~V}$ & $X$ & $\mathrm{~A}$ & $\mathrm{~A}$ \\
\hline 2 & TOA & & & A & $\mathrm{V}$ & $\bar{A}$ & $\overline{\mathrm{A}}$ & A & $\mathrm{O}$ & $X$ & $\mathrm{~V}$ & $\mathrm{~V}$ & $\mathrm{O}$ & $\mathrm{O}$ & $\mathrm{O}$ & $\mathrm{O}$ & $\mathrm{V}$ & $\mathrm{O}$ & $\mathrm{A}$ \\
\hline 3 & NIR & & & & $\mathrm{O}$ & $\mathrm{O}$ & $\mathrm{O}$ & $\mathrm{O}$ & $\mathrm{O}$ & $\mathrm{V}$ & $\mathrm{A}$ & $\mathrm{A}$ & $\mathrm{V}$ & $\mathrm{V}$ & $\mathrm{V}$ & $\mathrm{V}$ & $\mathrm{V}$ & $\mathrm{O}$ & $\mathrm{V}$ \\
\hline 4 & $\mathrm{CA}$ & & & & & A & $\mathrm{V}$ & $\mathrm{X}$ & $X$ & A & A & A & A & $\mathrm{O}$ & $\mathrm{O}$ & $\mathrm{O}$ & A & $\mathrm{A}$ & A \\
\hline 5 & NOR & & & & & & $\mathrm{V}$ & $\mathrm{V}$ & $\mathrm{V}$ & $\mathrm{V}$ & $\mathrm{O}$ & A & $\mathrm{V}$ & V & $\mathrm{V}$ & $\mathrm{V}$ & A & A & A \\
\hline 6 & RA & & & & & & & $\mathrm{X}$ & $\mathrm{X}$ & $\mathrm{A}$ & $\mathrm{A}$ & $\mathrm{A}$ & $\mathrm{A}$ & $\mathrm{O}$ & $\mathrm{O}$ & $\mathrm{O}$ & $\mathrm{A}$ & $\mathrm{A}$ & $\mathrm{A}$ \\
\hline 7 & ACD & & & & & & & & $\mathrm{O}$ & $\mathrm{X}$ & $\mathrm{V}$ & $\mathrm{V}$ & $\mathrm{V}$ & $\mathrm{V}$ & $\mathrm{V}$ & $\mathrm{V}$ & $\mathrm{V}$ & A & A \\
\hline 8 & SPS & & & & & & & & & $\mathrm{A}$ & $\mathrm{V}$ & $\mathrm{V}$ & $\mathrm{V}$ & $\mathrm{V}$ & $\mathrm{V}$ & $\mathrm{V}$ & $\mathrm{V}$ & $\mathrm{A}$ & $\mathrm{A}$ \\
\hline 9 & ASD & & & & & & & & & & A & A & A & V & $\mathrm{V}$ & $\mathrm{V}$ & V & A & A \\
\hline 10 & ES & & & & & & & & & & & $\mathrm{V}$ & $\mathrm{V}$ & $\mathrm{V}$ & $\mathrm{V}$ & $\mathrm{V}$ & $\mathrm{A}$ & $\mathrm{A}$ & $\mathrm{A}$ \\
\hline 11 & PSE & & & & & & & & & & & & $\mathrm{V}$ & $\mathrm{V}$ & $\mathrm{V}$ & $\mathrm{V}$ & $\mathrm{A}$ & $\mathrm{A}$ & $\mathrm{A}$ \\
\hline 12 & PTM & & & & & & & & & & & & & V & $\mathrm{V}$ & $\mathrm{V}$ & V & $\mathrm{A}$ & $\mathrm{V}$ \\
\hline 13 & RGDS & & & & & & & & & & & & & & $\mathrm{V}$ & V & A & A & A \\
\hline 14 & RGDe & & & & & & & & & & & & & & & V & A & A & A \\
\hline 15 & RGS & & & & & & & & & & & & & & & & A & A & A \\
\hline 16 & AHS & & & & & & & & & & & & & & & & & $\mathrm{A}$ & $\mathrm{A}$ \\
\hline 17 & $\mathrm{TC}$ & & & & & & & & & & & & & & & & & & V \\
\hline 18 & PWT & & & & & & & & & & & & & & & & & & \\
\hline
\end{tabular}

Fig 2: Initial reachability matrix

\begin{tabular}{|c|c|c|c|c|c|c|c|c|c|c|c|c|c|c|c|c|c|c|c|}
\hline S.NO. & & 1 & 2 & 3 & 4 & 5 & 6 & 7 & 8 & 9 & 10 & 11 & 12 & 13 & 14 & 15 & 16 & 17 & 19 \\
\hline & & NOP & $\begin{array}{l}\mathrm{T} \\
\mathrm{O} \\
\mathrm{A}\end{array}$ & NIR & $\begin{array}{l}\mathrm{C} \\
\mathrm{A}\end{array}$ & $\begin{array}{l}\mathrm{N} \\
\mathrm{O} \\
\mathrm{R}\end{array}$ & $\begin{array}{l}\mathrm{R} \\
\mathrm{A}\end{array}$ & $\begin{array}{l}A \\
C \\
D\end{array}$ & $\begin{array}{c}\text { SP } \\
\text { S }\end{array}$ & $\begin{array}{c}\text { AS } \\
\text { D }\end{array}$ & ES & $\begin{array}{c}\text { PS } \\
\text { E }\end{array}$ & $\begin{array}{l}\text { PT } \\
\text { M }\end{array}$ & $\begin{array}{c}\mathrm{R} \\
\mathrm{G} \\
\mathrm{DS}\end{array}$ & $\begin{array}{c}\mathrm{R} \\
\mathrm{G} \\
\mathrm{De}\end{array}$ & $\begin{array}{l}R \\
G \\
S\end{array}$ & $\begin{array}{l}\mathrm{A} \\
\mathrm{H} \\
\mathrm{S}\end{array}$ & TC & $\begin{array}{c}\mathrm{PW} \\
\mathrm{T}\end{array}$ \\
\hline 1 & NOP & 1 & 0 & 0 & 0 & 0 & 0 & 0 & 0 & 1 & 0 & 0 & 0 & 1 & 1 & 1 & 1 & 0 & 0 \\
\hline 2 & TOA & 1 & 1 & 0 & 1 & 0 & 0 & 0 & 0 & 0 & 1 & 1 & 0 & 0 & 0 & 0 & 1 & 0 & 0 \\
\hline 3 & NIR & 1 & 1 & 1 & 0 & 0 & 0 & 0 & 0 & 1 & 0 & 0 & 1 & 1 & 1 & 1 & 1 & 0 & 1 \\
\hline 4 & $\mathrm{CA}$ & 1 & 0 & 0 & 1 & 0 & 1 & 1 & 1 & 0 & 0 & 0 & 0 & 0 & 0 & 0 & 0 & 0 & 0 \\
\hline 5 & NOR & 1 & 1 & 0 & 1 & 1 & 1 & 1 & 1 & 1 & 0 & 0 & 1 & 1 & 1 & 1 & 0 & 0 & 0 \\
\hline 6 & RA & 1 & 1 & 0 & 0 & 0 & 1 & 1 & 1 & 0 & 0 & 0 & 0 & 0 & 0 & 0 & 0 & 0 & 0 \\
\hline 7 & ACD & 1 & 1 & 0 & 1 & 0 & 1 & 1 & 0 & 1 & 1 & 1 & 1 & 1 & 1 & 1 & 1 & 0 & 0 \\
\hline 8 & SPS & 0 & 0 & 0 & 1 & 0 & 1 & 1 & 1 & 0 & 1 & 1 & 1 & 1 & 1 & 1 & 1 & 0 & 0 \\
\hline 9 & ASD & 1 & 1 & 0 & 1 & 0 & 1 & 0 & 1 & 1 & 0 & 0 & 0 & 1 & 1 & 1 & 1 & 0 & 0 \\
\hline 10 & ES & 1 & 0 & 1 & 1 & 0 & 1 & 0 & 0 & 1 & 1 & 1 & 1 & 1 & 1 & 1 & 0 & 0 & 0 \\
\hline
\end{tabular}

$\mathrm{X}, \mathrm{O}$ by 1 or 0 as per the case. After incorporating the transitivity, the final reachability matrix is shown below in the Fig 3. 


\begin{tabular}{|c|c|c|c|c|c|c|c|c|c|c|c|c|c|c|c|c|c|c|c|}
\hline 11 & PSE & 1 & 0 & 1 & 1 & 1 & 1 & 0 & 0 & 1 & 0 & 1 & 1 & 1 & 1 & 1 & 0 & 0 & 0 \\
\hline 12 & PTM & 1 & 0 & 0 & 1 & 0 & 1 & 0 & 0 & 1 & 0 & 0 & 1 & 1 & 1 & 1 & 1 & 0 & 1 \\
\hline 13 & RGDS & 0 & 0 & 0 & 0 & 0 & 0 & 0 & 0 & 0 & 0 & 0 & 0 & 1 & 1 & 1 & 0 & 0 & 0 \\
\hline 14 & RGDe & 0 & 0 & 0 & 0 & 0 & 0 & 0 & 0 & 0 & 0 & 0 & 0 & 0 & 1 & 1 & 0 & 0 & 0 \\
\hline 15 & RGS & 0 & 0 & 0 & 0 & 0 & 0 & 0 & 0 & 0 & 0 & 0 & 0 & 0 & 0 & 1 & 0 & 0 & 0 \\
\hline 16 & AHS & 1 & 0 & 0 & 1 & 1 & 1 & 0 & 0 & 0 & 1 & 1 & 0 & 1 & 1 & 1 & 1 & 0 & 0 \\
\hline 17 & TC & 1 & 0 & 0 & 1 & 1 & 1 & 1 & 1 & 1 & 1 & 1 & 1 & 1 & 1 & 1 & 1 & 1 & 1 \\
\hline 18 & PWT & 1 & 1 & 0 & 1 & 1 & 1 & 1 & 1 & 1 & 1 & 1 & 0 & 1 & 1 & 1 & 1 & 0 & 1 \\
\hline
\end{tabular}

Fig 3 : Final reachability matrix

\begin{tabular}{|c|c|c|c|c|c|c|c|c|c|c|c|c|c|c|c|c|c|c|c|c|}
\hline S.NO. & & 1 & 2 & 3 & 4 & 5 & 6 & 7 & 8 & 9 & 10 & 11 & 12 & 13 & 14 & 15 & 16 & 17 & 18 & D.P \\
\hline & & NOP & $\begin{array}{l}\mathrm{T} \\
\mathrm{O} \\
\mathrm{A}\end{array}$ & NIR & $\begin{array}{l}\mathrm{C} \\
\mathrm{A}\end{array}$ & $\begin{array}{l}\mathrm{N} \\
\mathrm{O} \\
\mathrm{R}\end{array}$ & $\begin{array}{l}\mathrm{R} \\
\mathrm{A}\end{array}$ & $\begin{array}{l}\text { A } \\
\text { C } \\
\text { D }\end{array}$ & $\begin{array}{c}\text { SP } \\
\text { S }\end{array}$ & $\begin{array}{c}\text { AS } \\
\text { D }\end{array}$ & ES & $\begin{array}{c}\text { PS } \\
\text { E }\end{array}$ & $\begin{array}{l}\text { PT } \\
\text { M }\end{array}$ & $\begin{array}{c}\mathrm{R} \\
\mathrm{G} \\
\mathrm{DS}\end{array}$ & $\begin{array}{c}R \\
G \\
\text { De }\end{array}$ & $\begin{array}{l}\mathrm{R} \\
\mathrm{G} \\
\mathrm{S}\end{array}$ & $\begin{array}{l}\mathrm{A} \\
\mathrm{H} \\
\mathrm{S}\end{array}$ & $\begin{array}{l}\mathrm{T} \\
\mathrm{C}\end{array}$ & $\begin{array}{c}\mathrm{P} \\
\mathrm{W} \\
\mathrm{T}\end{array}$ & \\
\hline 1 & NOP & 1 & 1 & 0 & 1 & 1 & 1 & 0 & 1 & 1 & 1 & 1 & 0 & 1 & 1 & 1 & 1 & 0 & 0 & 13 \\
\hline 2 & TOA & 1 & 1 & 1 & 1 & 1 & 1 & 0 & 1 & 1 & 1 & 1 & 0 & 1 & 1 & 1 & 1 & 0 & 0 & 14 \\
\hline 3 & NIR & 1 & 1 & 1 & 1 & 0 & 1 & 0 & 1 & 1 & 1 & 1 & 1 & 1 & 1 & 1 & 1 & 0 & 1 & 15 \\
\hline 4 & $\mathrm{CA}$ & 1 & 1 & 0 & 1 & 0 & 1 & 1 & 1 & 1 & 1 & 1 & 1 & 1 & 1 & 1 & 1 & 0 & 0 & 14 \\
\hline 5 & NOR & 1 & 1 & 0 & 1 & 1 & 1 & 1 & 1 & 1 & 1 & 1 & 1 & 1 & 1 & 1 & 0 & 0 & 0 & 14 \\
\hline 6 & RA & 1 & 1 & 1 & 1 & 0 & 1 & 1 & 1 & 1 & 1 & 1 & 0 & 1 & 1 & 1 & 1 & 0 & 0 & 14 \\
\hline 7 & ACD & 1 & 1 & 1 & 1 & 0 & 1 & 1 & 0 & 1 & 1 & 1 & 1 & 1 & 1 & 1 & 1 & 0 & 0 & 14 \\
\hline 8 & SPS & 1 & 1 & 0 & 1 & 0 & 1 & 1 & 1 & 1 & 1 & 1 & 1 & 1 & 1 & 1 & 1 & 0 & 0 & 14 \\
\hline 9 & ASD & 1 & 1 & 0 & 1 & 0 & 1 & 0 & 1 & 1 & 1 & 1 & 1 & 1 & 1 & 1 & 1 & 0 & 0 & 14 \\
\hline 10 & ES & 1 & 1 & 1 & 1 & 0 & 1 & 1 & 1 & 1 & 1 & 1 & 1 & 1 & 1 & 1 & 1 & 0 & 0 & 15 \\
\hline 11 & PSE & 1 & 1 & 1 & 1 & 1 & 1 & 1 & 1 & 1 & 0 & 1 & 1 & 1 & 1 & 1 & 1 & 0 & 0 & 15 \\
\hline 12 & PTM & 1 & 1 & 0 & 1 & 0 & 1 & 1 & 1 & 1 & 1 & 1 & 1 & 1 & 1 & 1 & 1 & 0 & 1 & 14 \\
\hline 13 & RGDS & 0 & 0 & 0 & 0 & 0 & 0 & 0 & 0 & 0 & 0 & 0 & 0 & 1 & 1 & 1 & 0 & 0 & 0 & 03 \\
\hline 14 & RGDe & 0 & 0 & 0 & 0 & 0 & 0 & 0 & 0 & 0 & 0 & 0 & 0 & 0 & 1 & 1 & 0 & 0 & 0 & 02 \\
\hline 15 & RGS & 0 & 0 & 0 & 0 & 0 & 0 & 0 & 0 & 0 & 0 & 0 & 0 & 0 & 0 & 1 & 0 & 0 & 0 & 01 \\
\hline 16 & AHS & 1 & 1 & 0 & 1 & 1 & 1 & 0 & 1 & 1 & 1 & 1 & 0 & 1 & 1 & 1 & 1 & 0 & 0 & 13 \\
\hline 17 & TC & 1 & 1 & 0 & 1 & 1 & 1 & 1 & 1 & 1 & 1 & 1 & 1 & 1 & 1 & 1 & 1 & 1 & 1 & 17 \\
\hline 18 & PWT & 1 & 1 & 0 & 1 & 1 & 1 & 1 & 1 & 1 & 1 & 1 & 1 & 1 & 1 & 1 & 1 & 0 & 1 & 16 \\
\hline De.P. & & 15 & 15 & 06 & 15 & 07 & 15 & 10 & 14 & 15 & 14 & 15 & 11 & 16 & 17 & 18 & 14 & 01 & 04 & \\
\hline
\end{tabular}




\subsection{Level Partition}

Table 2 : Iteration I

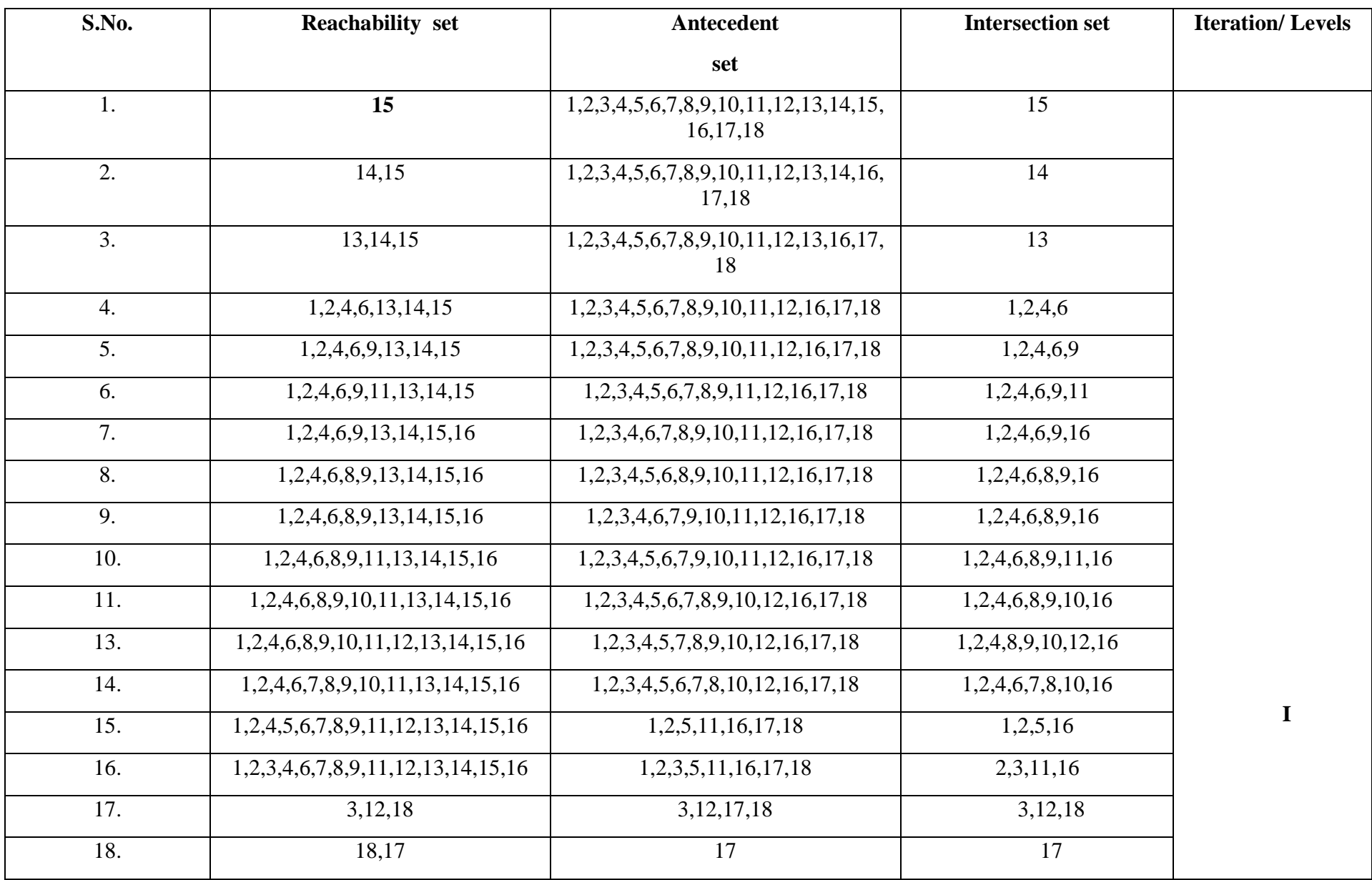

Table 3 : Iteration II

\begin{tabular}{|c|c|c|c|c|}
\hline S.No. & Reachability set & Antecedent set & Intersection set & Iteration \\
\hline 2. & 14 & $\begin{array}{c}1,2,3,4,5,6,7,8,9,10,11,12,13,14,16 \\
17,18\end{array}$ & 14 & II \\
\hline 3. & 13,14 & $\begin{array}{c}1,2,3,4,5,6,7,8,9,10,11,12,13,16,17 \\
18\end{array}$ & 13 & \\
\hline 4. & $1,2,4,6,13,14$ & $1,2,3,4,5,6,7,8,9,10,11,12,16,17,18$ & $1,2,4,6$ & \\
\hline 5. & $1,2,4,6,9,13,14$ & $1,2,3,4,5,6,7,8,9,10,11,12,16,17,18$ & $1,2,4,6,9$ & \\
\hline 6. & $1,2,4,6,9,11,13,14$ & $1,2,3,4,5,6,7,8,9,11,12,16,17,18$ & $1,2,4,6,9,11$ & \\
\hline 7. & $1,2,4,6,9,13,14,16$ & $1,2,3,4,6,7,8,9,10,11,12,16,17,18$ & $1,2,4,6,9,16$ & \\
\hline 8. & $1,2,4,6,8,9,13,14,16$ & $1,2,3,4,5,6,8,9,10,11,12,16,17,18$ & $1,2,4,6,8,9,16$ & \\
\hline 9. & $1,2,4,6,8,9,13,14,16$ & $1,2,3,4,5,6,7,9,10,11,12,16,17,18$ & $1,2,4,6,8,9,16$ & \\
\hline 10. & $1,2,4,6,8,9,11,13,14,16$ & $1,2,4,5,6,7,9,10,11,12,16,17,18$ & $1,2,4,6,8,9,11,16$ & \\
\hline 11. & $1,2,4,6,8,9,10,11,13,14,16$ & $1,2,3,4,5,6,7,8,9,10,12,16,17,18$ & $1,2,4,6,8,9,10,16$ & \\
\hline 13. & $\begin{array}{c}1,2,4,6,8,9,10,11,12,13,14,1 \\
6\end{array}$ & $1,2,3,4,5,7,8,9,10,12,16,17,18$ & $1,2,4,8,9,10,12,16$ & \\
\hline 14. & $1,2,4,6,7,8,9,10,11,13,14,16$ & $1,2,3,4,5,6,7,8,10,12,16,17,18$ & $1,2,4,6,7,8,10,16$ & \\
\hline 15. & $1,2,4,5,6,8,9,11,12,13,14,16$ & $1,2,5,11,16,17,18$ & $1,2,5,16$ & \\
\hline
\end{tabular}




\begin{tabular}{|c|c|c|c|}
\hline 16. & $1,2,3,4,6,8,9,11,12,13,14,16$ & $1,2,3,5,11,16,17,18$ & $1,2,3,11,16$ \\
\hline 17. & $3,12,18$ & $3,12,17,18$ & $3,12,18$ \\
\hline 18. & 18,17 & 17 & 17 \\
\hline
\end{tabular}

Table 4 : Iteration III

\begin{tabular}{|c|c|c|c|c|}
\hline $\begin{array}{l}\text { Sr. } \\
\text { No. }\end{array}$ & $\begin{array}{c}\text { Reachability } \\
\text { set }\end{array}$ & Antecedent set & $\begin{array}{l}\text { Intersectio } \\
\text { n set }\end{array}$ & $\begin{array}{l}\text { Itera } \\
\text { tion }\end{array}$ \\
\hline 3. & 13 & $\begin{array}{c}1,2,3,4,5,6,7,8,9 \\
10,11,12,13,16,17 \\
18\end{array}$ & 13 & \\
\hline 4. & $1,2,4,6,13$ & $\begin{array}{c}1,2,3,4,5,6,7,8,9,10 \\
, 11,12,16,17,18\end{array}$ & $1,2,4,6$ & \\
\hline 5. & $1,2,4,6,9,13$ & $\begin{array}{c}1,2,3,4,5,6,7,8,9, \\
10,11,12,16,17,18\end{array}$ & $1,2,4,6,9$ & \\
\hline 6. & $\begin{array}{c}1,2,4,6,9,11 \\
13\end{array}$ & $\begin{array}{c}1,2,3,4,5,6,7,8,9 \\
11,12,16,17,18\end{array}$ & $\begin{array}{c}1,2,4,6,9 \\
11\end{array}$ & III \\
\hline 7. & $\begin{array}{c}1,2,4,6,9,13 \\
16\end{array}$ & $\begin{array}{c}1,2,3,4,5,6,7,8,9, \\
10,11,12,16,17,18\end{array}$ & $\begin{array}{c}1,2,4,6,9 \\
16\end{array}$ & \\
\hline 8. & $\begin{array}{c}1,2,4,6,8,9 \\
13,16\end{array}$ & $\begin{array}{c}1,2,3,4,5,6,8,9,10,1 \\
1,12,16,17,18\end{array}$ & $\begin{array}{c}1,2,4,6,8,9 \\
16\end{array}$ & \\
\hline 9. & $\begin{array}{c}1,2,4,6,8,9 \\
13,16\end{array}$ & $\begin{array}{c}1,2,3,4,5,6,7,9,10,1 \\
1,12,16,17,18\end{array}$ & $\begin{array}{c}1,2,4,6,8,9 \\
16\end{array}$ & \\
\hline 10. & $\begin{array}{c}1,2,4,6,8,9,1 \\
1,13,16\end{array}$ & $\begin{array}{c}1,2,3,4,5,6,7,9,10,1 \\
1,12,16,17,18\end{array}$ & $\begin{array}{c}1,2,4,6,8,9 \\
11,16\end{array}$ & \\
\hline 11. & $\begin{array}{l}1,2,4,6,8,9 \\
10,11,13,16\end{array}$ & $\begin{array}{c}1,2,3,4,5,6,7,8,9 \\
10,12,16,17,18\end{array}$ & $\begin{array}{c}1,2,4,6,8,9 \\
10,16\end{array}$ & \\
\hline 13. & $\begin{array}{c}1,2,4,6,8,9 \\
10,11,12,13 \\
16\end{array}$ & $\begin{array}{c}1,2,3,4,5,7,8,9,10,1 \\
2,16,17,18\end{array}$ & $\begin{array}{l}1,2,4,8,9, \\
10,12,16\end{array}$ & \\
\hline 14. & $\begin{array}{c}1,2,4,6,7,8,9 \\
10,11,13,16\end{array}$ & $\begin{array}{c}1,2,3,4,5,6,7,8,10,1 \\
2,16,17,18\end{array}$ & $\begin{array}{c}1,2,4,6,7,8 \\
10,16\end{array}$ & \\
\hline 15. & $\begin{array}{c}1,2,4,5,6,8,9 \\
11,12,13,16\end{array}$ & $1,2,5,11,16,17,18$ & $1,2,5,16$ & \\
\hline 16. & $\begin{array}{c}1,2,3,4,6,8,9 \\
11,12,13,16\end{array}$ & $1,2,3,11,16,17,18$ & $2,3,11,16$ & \\
\hline 17. & $3,12,18$ & $3,12,17,18$ & $3,12,18$ & \\
\hline 18. & 18,17 & 17 & 17 & \\
\hline
\end{tabular}

Table 5 : Iteration IV

\begin{tabular}{|c|c|c|c|c|}
\hline $\begin{array}{c}\text { S. } \\
\text { No } \\
\cdot\end{array}$ & $\begin{array}{c}\text { Reachability } \\
\text { set }\end{array}$ & Antecedent set & $\begin{array}{c}\text { Intersect } \\
\text { ion set }\end{array}$ & $\begin{array}{c}\text { Iteratio } \\
\mathbf{n} / \\
\text { Levels }\end{array}$ \\
\cline { 1 - 3 } & $1,2,4,6$ & $1,2,3,4,5,6,7,8,9$, & $1,2,4,6$ & IV \\
& & $10,11,12,16,17,18$ & & \\
\cline { 1 - 2 }. & $\mathbf{1 , 2 , 4 , 6 , 9}$ & $1,2,3,4,5,6,7,8,9$, & $\mathbf{1 , 2 , 4 , 6 , 9}$ & \\
\hline
\end{tabular}

\begin{tabular}{|c|c|c|c|}
\hline & & $10,11,12,16,17,18$ & \\
\hline 6. & $1,2,4,6,9,11$ & $\begin{array}{c}1,2,3,4,5,6,7,8,9 \\
11,12,16,17,18\end{array}$ & $\begin{array}{c}1,2,4,6,9, \\
11\end{array}$ \\
\hline 7. & $1,2,4,6,9,16$ & $\begin{array}{c}1,2,3,4,5,6,7,8,9 \\
10,11,12,16,17,18\end{array}$ & $\begin{array}{c}1,2,4,6,9, \\
16\end{array}$ \\
\hline 8. & $\begin{array}{c}1,2,4,6,8,9 \\
16\end{array}$ & $\begin{array}{c}1,2,3,4,5,6,8,9,10,1 \\
1,12,16,17,18\end{array}$ & $\begin{array}{c}1,2,4,6,8 \\
9,16\end{array}$ \\
\hline 9. & $\begin{array}{c}1,2,4,6,8,9 \\
16\end{array}$ & $\begin{array}{c}1,2,3,4,6,7,9,10 \\
11,12,16,17,18\end{array}$ & $\begin{array}{c}1,2,4,6,8 \\
9,16\end{array}$ \\
\hline 10. & $\begin{array}{c}1,2,4,6,8,9 \\
11,16\end{array}$ & $\begin{array}{c}1,2,3,4,5,6,7,9,11,1 \\
2,16,17,18\end{array}$ & $\begin{array}{c}1,2,4,6,8 \\
9,11,16\end{array}$ \\
\hline 11. & $\begin{array}{c}1,2,4,6,8,9 \\
10,11,16\end{array}$ & $\begin{array}{c}1,2,3,4,5,6,7,8,9, \\
10,12,16,17,18\end{array}$ & $\begin{array}{c}1,2,4,6,8 \\
9,10,16\end{array}$ \\
\hline 13. & $\begin{array}{l}1,2,4,6,8,9 \\
10,11,12,16\end{array}$ & $\begin{array}{c}1,2,3,4,5,7,8,9,10,1 \\
2,16,17,18\end{array}$ & $\begin{array}{c}1,2,4,8,9 \\
10,12 \\
16\end{array}$ \\
\hline 14. & $\begin{array}{c}1,2,4,6,7,8,9 \\
10,11,16\end{array}$ & $\begin{array}{c}1,2,4,5,6,7,8,10 \\
12,16,17,18\end{array}$ & $\begin{array}{c}1,2,4,6,7 \\
8,10,16\end{array}$ \\
\hline 15. & $\begin{array}{c}1,2,4,5,6,8,9 \\
11,12,16\end{array}$ & $1,2,5,11,16,17,18$ & $1,2,5,16$ \\
\hline 16. & $\begin{array}{c}1,2,3,4,6,8,9 \\
11,12,16\end{array}$ & $\begin{array}{c}1,2,3,5,11,16,17 \\
18\end{array}$ & $\begin{array}{c}2,3,11 \\
16\end{array}$ \\
\hline 17. & $3,12,18$ & $3,12,17,18$ & $3,12,18$ \\
\hline
\end{tabular}

Table 6 : Iteration V

\begin{tabular}{|c|c|c|c|c|}
\hline $\begin{array}{l}\text { Sr. } \\
\text { No. }\end{array}$ & $\begin{array}{c}\text { Reachability } \\
\text { set }\end{array}$ & $\begin{array}{c}\text { Antecedent } \\
\text { set }\end{array}$ & $\begin{array}{c}\text { Intersection } \\
\text { set }\end{array}$ & $\begin{array}{l}\text { Itera } \\
\text { tion }\end{array}$ \\
\hline 6. & 11 & $\begin{array}{c}5,7,8,11,12,16 \\
17,18\end{array}$ & 11 & \\
\hline 7. & 16 & $\begin{array}{c}3,7,8,10,11,12 \\
16,17,18\end{array}$ & 16 & \\
\hline 8. & 8,16 & $\begin{array}{c}3,5,8,10,11,12 \\
16,17,18\end{array}$ & 8,16 & \\
\hline 9. & 8,16 & $\begin{array}{c}3,7,10,11,12,16 \\
, 17,18\end{array}$ & 8,16 & \\
\hline 10. & $8,11,16$ & $\begin{array}{c}3,7,10,11,12,16 \\
, 17,18\end{array}$ & $8,11,16$ & \\
\hline 11. & $8,10,11,16$ & $\begin{array}{c}3,5,7,8,10,12,1 \\
6,17,18\end{array}$ & $3,8,10,16$ & \\
\hline 13. & $\begin{array}{c}8,10,11,12,1 \\
6\end{array}$ & $\begin{array}{c}3,5,7,8,10,12,1 \\
6,17,18\end{array}$ & $3,8,10,12,16$ & \\
\hline
\end{tabular}




\begin{tabular}{|c|c|c|c|c|}
\hline 14. & $7,8,10,11,16$ & $\begin{array}{c}7,8,10,12,16,17 \\
, 18\end{array}$ & $7,8,10,16$ & $\mathbf{V}$ \\
\hline 15. & $5,8,11,12,16$ & $5,11,16,17,18$ & 5,16 & \\
\hline 16. & $3,8,11,12,16$ & $3,11,16,17,18$ & $3,11,16$ & \\
\hline 17. & $3,12,18$ & $3,12,17,18$ & $3,12,18$ & \\
\hline 18. & 18,17 & 17 & 17 & \\
\hline
\end{tabular}

Table 8 : Iteration VI

\begin{tabular}{|c|c|c|c|c|}
\hline $\begin{array}{l}\text { Sr. } \\
\text { No. }\end{array}$ & $\begin{array}{c}\text { Reachability } \\
\text { set }\end{array}$ & Antecedent set & $\begin{array}{c}\text { Intersection } \\
\text { set }\end{array}$ & $\begin{array}{l}\text { Itera } \\
\text { tion }\end{array}$ \\
\hline 11. & 10 & $\begin{array}{c}3,5,7,10,12,17 \\
18\end{array}$ & 10 & \\
\hline 13. & 10,12 & $\begin{array}{c}3,5,7,10,12,17 \\
18\end{array}$ & 10,12 & \\
\hline 14. & 7,10 & $\begin{array}{c}3,5,7,10,12,17 \\
18\end{array}$ & 7,10 & \\
\hline 15. & 5,12 & $5,17,18$ & 5 & \\
\hline 16. & 3,12 & $3,17,18$ & 3 & VI \\
\hline 17. & $3,12,18$ & $3,12,17,18$ & $3,12,18$ & \\
\hline 18. & 18,17 & 17 & 17 & \\
\hline
\end{tabular}

Table 9 : Iteration VII

\begin{tabular}{|c|c|c|c|c|}
\hline $\begin{array}{c}\text { Sr. } \\
\text { No. }\end{array}$ & $\begin{array}{c}\text { Reachability } \\
\text { set }\end{array}$ & $\begin{array}{c}\text { Antecedent } \\
\text { set }\end{array}$ & $\begin{array}{c}\text { Intersection } \\
\text { set }\end{array}$ & $\begin{array}{c}\text { Itera } \\
\text { tion }\end{array}$ \\
\hline 15. & $\mathbf{5}$ & $5,17,18$ & 5 & \\
\cline { 2 - 4 } 16. & 3 & $3,17,18$ & 3 & \multirow{2}{*}{ VII } \\
\hline 17. & $\mathbf{3 , 1 8}$ & $3,17,18$ & 3,18 & \\
\hline 18. & 18,17 & 17 & 17 & \\
\hline
\end{tabular}

Table 10 : Iteration VIII

\begin{tabular}{|c|c|c|c|c|}
\hline $\begin{array}{c}\text { Sr. } \\
\text { No. }\end{array}$ & $\begin{array}{c}\text { Reachability } \\
\text { set }\end{array}$ & $\begin{array}{c}\text { Antecedent } \\
\text { set }\end{array}$ & $\begin{array}{c}\text { Intersection } \\
\text { set }\end{array}$ & $\begin{array}{c}\text { Itera } \\
\text { tion }\end{array}$ \\
\hline 18. & $\mathbf{1 7}$ & 17 & 17 & \\
& & & & VIII \\
\hline
\end{tabular}

\subsection{Classification of factors}

The critical success factors described earlier are classified in to four clusters viz. autonomous factor, dependent factors, linkage factors and independent factors (mentioned in Table XII below). Fig. 4 below shows the driving power and dominance diagram

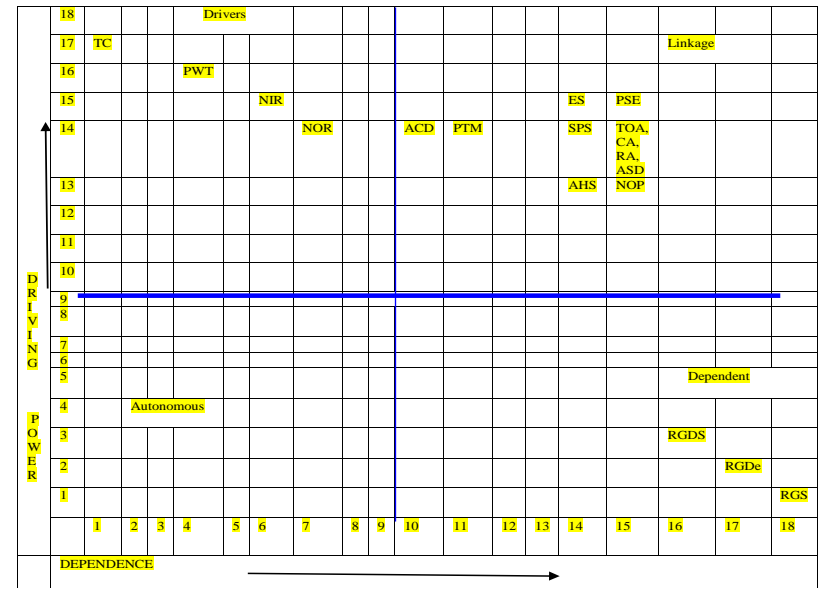

Fig . 4: Driving power and dependence diagram

\subsection{ISM Model}

An ISM model is developed ( as shown in fig. 5 below ) after arranging the elements as per their interaction or dependence relationships.

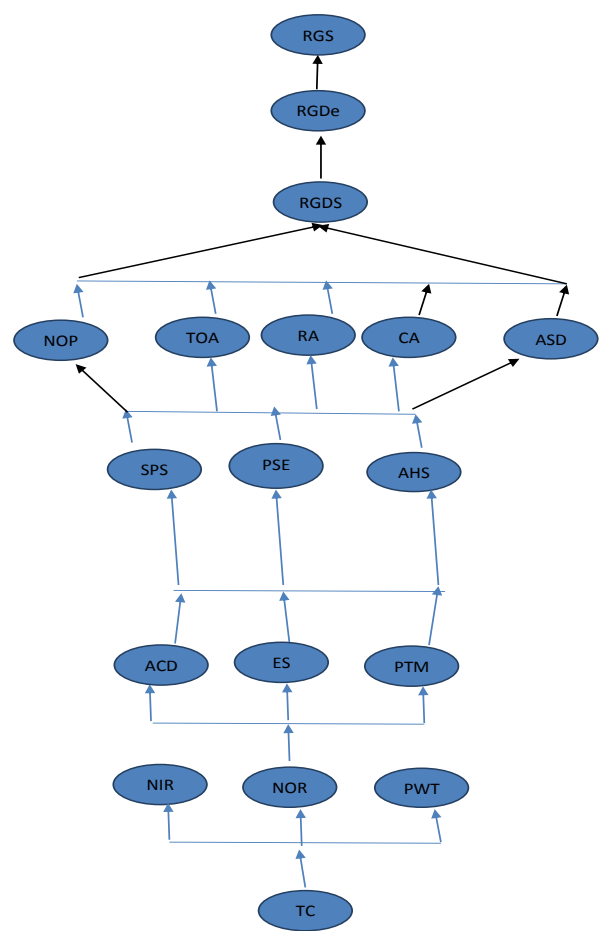

Fig 5: ISM diagraph

\section{CONCLUSIONS AND MANAGERIAL IMPLICATIONS}

1. Use of health care dashboards : Now days health care centers also make use of health care dashboards. These dashboards are customized as per customer's need and are $100 \%$ HIPAA compliant. They are primarily used to track healthcare information data, KPIs and other essential metrics. These are further used to analyze the data of a particular healthcare department, a specific medical process in a hospital, or a private clinic. Through this analysis, management can standardized the processes across the practice to reduce variations in health care delivery experience and further used to improve the ability to identify the trends and patterns and accordingly make necessary business changes. 
2. Handling huge and valuable data : The sheer amount of data generated by a business as complex as a hospital or medical treatment facility can be staggeringly overwhelming. So, it is quite mandatory now days to closely monitor the data and measure the appropriate metrics that can improve the overall health of the organization, the metrics which can generate more revenue for the facility and provide more financial opportunities for the health acre facility.

\section{ACKNOWLEDGEMENT}

Authors are thankful to Prof. S.P Singh, Department of Management Studies for imparting knowledge on ISM methodology which helped authors substantially while writing this piece of work.

\section{REFERENCES}

[1] https://www.datapine.com/kpi-examples-andtemplates/healthcare
[2] https://kaysharbor.com/blog/healthcare/5-healthcaredashboard-metrics

[3] Manoj Mohanan, Katherine Hay and Nachiket Mor , Quality of health care in India : Challenges, priorities and the road ahead https://www.healthaffairs.org/doi/full/10.1377hlthaff.201 6.0676

[4] Gupta , I. and Bhatia , M. (2016) . The Indian health care system. The commonwealth fund -International health care system profiles. $\mathrm{http} / / /$ internationalcommonwealthfund.org/countries/Indi a

[5] Reimagining the possible in the Indian healthcare ecosystem with emerging technologies, the Bengal chamber report , 2018 , www.pwc.in.

[6] Warfield, J., N. 1974. Developing interconnection matrices in structural modeling. IEEE Transactions on System, Man, and Cybernetics (SMC) .4 (1), 81-87. 